\title{
Keratosis Pilaris Rubra Totalis: Clinical and Histopathological Study with New insights
}

\author{
${ }^{1}$ Professor Khalifa E. Sharquie MD; PhD; ${ }^{1}$ Professor Adil A. Noaimi MD; FICMS.; ${ }^{2}$ Dr. Raed I. \\ Jabbar MD; CABD.
}

${ }^{1}$ Department of Dermatology, College of Medicine, University of Baghdad. Iraqi and Arab Board of Dermatology and Venereology, Center of Dermatology and Venereology, Medical City, Baghdad, Iraq. ${ }^{2}$ Center of Dermatology and Venereology, Medical City, Baghdad, Iraq

\section{ABSTRACT}

Background: Keratosis pilaris rubra (KPR) is a common but unrecognized follicular keratinizatioin. It is a common skin problem among Iraqi population and most cases were misdiagnosed as dermatitis as there are few reports in medical literature. Objective: To do full clinical and histopathological evaluation of KPR in Iraqi population.Patients and Methods: This is case descriptive, clinico-histopathological outpatient based study. It was done in the Center of Dermatology and Venereology, Baghdad Teaching Hospital, Medical City, Iraq during the period from March 2016 to October 2017. Sixty patients were enrolled in this study. History and dermatological examination were carried out for all patients. Skin biopsy was done for 10 patients for histopathological study using Hematoxylin and Eosin stain. Results: A total of the 60 patients were diagnosed as KPR during the study period. Thirty nine $(65 \%)$ males and $21(35 \%)$ females. The age at onset of disease ranged from since birth to $8(2.9 \pm 2.17)$ years . Facial involvement included the cheeks in 60 (100\%) patients. Other sites of involvement included the arms where the lateral aspects involved in $60(100 \%)$ patients and the medial aspect $11(18.3 \%)$, thighs including the buttocks in 51 (85\%), trunk 25 (41.6\%), legs $22(36.6 \%)$ and the neck $15(25 \%)$.The rash was erythematous all over but most obvious on the sides of face presenting as red, rough face. Histopathological evaluation showed follicular plugging in (100\%) with dilatation of both superficial and deep vascular plexus in $(75 \%)$ of cases. Limitation: The main limitation of this study was the lack of follow up data related to the subject of the study. Conclusion: KPR is a common condition among Iraqi population and this study is the largest study carried out all over the world. As most areas of body are involved, the name keratosis pilaris rubra totalis is suggested.

Keywords: Keratosis pilaris rubra, follicular papules, red face.

*Correspondence to Author: Professor Khalifa E. Sharquie Department of Dermatology, College of Medicine, University of Baghdad Iraqi and Arab Board of Dermatology and Venereology, Center of Dermatology and Venereology, Baghdad Teaching Hospital, Medical City, Medical Collection Office, P.O. BOX 61080 Postal code 12114, Baghdad, Iraq

\section{How to cite this article:}

Khalifa E. Sharquie, Adil A. Noaimi, Raed I. Jabbar. Keratosis Pilaris Rubra Totalis: Clinical and Histopathological Study with New insights. American Journal of Dermatological Research and Reviews, $2020,3: 25$

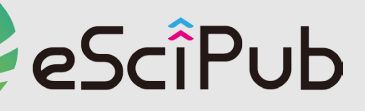

eSciPub LLC, Houston, TX USA. Website: https://escipub.com/ 


\section{Introduction}

Keratosis pilaris $(K P)$ is an inherited abnormality of keratinization affecting the follicular orifices with varying degrees of keratotic follicular plugging, perifollicular erythema and follicular atrophy. It is a common skin condition and can be considered a variant of normal rather than a disease, as up to half of the population present with the condition. 1,2

Incidence and prevalence: $\mathrm{KP}$ is a very common condition affecting $50-80 \%$ of adolescents and about $40 \%$ of adults. It often presents in the first decade of life and may worsen around puberty. In some patients, the disorder improves with age but any age group can be affected from childhood to old age. Females appear to be more frequently affected than males and it is seen in all ethnic groups ${ }^{3}$

Keratosis pilaris rubra: Erythema is often present in typical KP; it is usually mild and limited to the perifollicular skin. When perifollicular erythema is more noticeable, the disorder has been called keratosis pilaris rubra (KPR). These findings are usually limited to the cheeks, forehead, and neck. ${ }^{4}$

KPR seems to be relatively common but uncommonly reported condition .It is characterized by substantial erythema, wide spread involvement and persistent after the onset of puberty but without the atrophy or hyperpigmentation noted in certain keratosis pilaris variants. The erythematous component of KPR persist or even worsen at puberty, without progression to scarring. As with other form of $\mathrm{KP}$, the pathogenesis of KPR is not well understood .The erythema present in KPR fluctuates and in some patients is present even in areas without significant keratotic papules .This raises the question of whether flushing via autonomic dysregulation may have role in clinical manifestation. ${ }^{5}$

So the aim of the present work is to do full clinical and histopathological evaluation of keratosis pilaris rubra in Iraqi population.

\section{Patients and Methods}

This is case descriptive, clinical and histopathological study accomplished at the Center of Dermatology and Venereology, Baghdad Teaching Hospital/ Medical City, Iraq during the period from March 2016 to October 2017.

Formal consent was taken from each patient and/or their parents before starting the study. The aim of the study and the nature, course and prognosis of the disease in addition to available treatment options were fully explained and discussed with the participants and/ or their relatives. Also, the ethical approval was given by the scientific committee of the Scientific Council of Dermatology and Venereology-Arab Board for Health Specializations. Digital photography was taken using Sony digital, 16.1 megapixel camera in the same place and distance with fixed illumination.

The diagnosis was depended on history and clinical basis. Detailed history was taken from each patient and/ or their parents regarding: age of onset and age at presentation, gender, chief compliant, other skin diseases, family history of the same disease and past medical and drug history.

Full clinical examination was done including the site and clinical appearance of the lesion. Skin biopsy was done for 10 patients from the most characterized skin lesions at different sites and sent for histopathological examination using Hematoxylin and Eosin stain (H\&E stain).

Sixty patients were enrolled in the study; many of these cases were misdiagnosed and treated as dermatitis especially in infancy and childhood.

Data were statistically described in terms of range, mean, standard deviation ( \pm SD), frequencies (no. of cases) and relative frequencies (percentage). All statistical calculation were done using computer statistical program SPSS version 23. 


\section{Results}

\section{Clinical results:}

A total of the 60 patients were diagnosed as Keratosis pilaris rubra during the study period.

Thirty nine (65\%) males and $21(35 \%)$ females with male to female ratio 1.6:1. The age at onset of disease ranged from since birth to 8 years with a mean $\pm S D$ of $2.9 \pm 2.18$ years. Their ages at presentation ranged from 6 months to 36 years with a mean $\pm S D$ of $13.6 \pm 8.29$ years.

In most patients the eruption was asymptomatic, although $8(13,3 \%)$ patients noticed occasional itching. Other family member with KPR was positive in $8(13,33 \%)$ patients. All patients suffered from facial erythematous patch with illdefined border. (Figure 1)

Marked erythema with follicular prominence was noticed in all patients most commonly affecting the cheeks and lateral aspect of the proximal extremities. (Figures $2 \& 3$ ).

The rash was erythematous all over but most obvious on the sides of face presenting as red, rough face without scarring or hair loss,. The erythema of the face in 14 (23.33\%) patients waxed and waned but never completely resolved, while $46(76.66 \%)$ patients showed constant erythema.

Facial involvement included the cheeks in 60 $(100 \%)$ patients, the eye brows $10(16.5 \%)$, the forehead $7(11.6 \%)$, the chin $5(8.3 \%)$ and the glabella $5(8.3 \%)$.Patients could be presented with diffuse facial rash with redness as a result of coalescence of the red follicular papules giving appearance of red cheeks, but in many patients the rash was generalized (Figure 4).

All lesions were follicular keratotic papules usually erythematous in color and in some areas these follicular erythematous papules coalesce together to form red patches and this typically was noticed in the face giving a butterfly appearance. (Figure 5)

Distribution of the lesions according to the site are showing in table 1.

History of asthma and allergic rhinitis was observed in 8 (13.33) and $6(10 \%)$ patients respectively. No other chronic illness were noticed.

\section{Histopathological results:}

Light microscopic examination of H\&E stained sections showed the following findings: (Figures 6-8)

- Basket weave appearance of stratum corneum in 5 (50\%) cases.

- Epidermal psoriasiform hyperplasia in 3 (30\%).

- Keratin plug fills and dilates the infundibulum of the hair follicle in 10 $(100 \%)$.

- Mild superficial and deep perivascular lymphocytic infiltrates in 4 (40\%).

- Mild dilatation of both superficial and deep vascular plexus in 7 (70\%).

- Intrafollicular lymphocytic infiltrates in 2 $(20 \%)$.

Table 1. Distribution of the lesions according to the site.

\begin{tabular}{lll}
\hline Site of involvement & Number of patients & Percentage \\
\hline Face & 60 & 100 \\
\hline Arms: & 60 & 100 \\
Lateral aspect & 11 & 18.3 \\
Medial aspect & 51 & 85 \\
\hline Thighs including & 25 & 41.6 \\
the buttocks & 22 & 36.6 \\
\hline Trunk & 15 & 25 \\
\hline Legs & Neck &
\end{tabular}




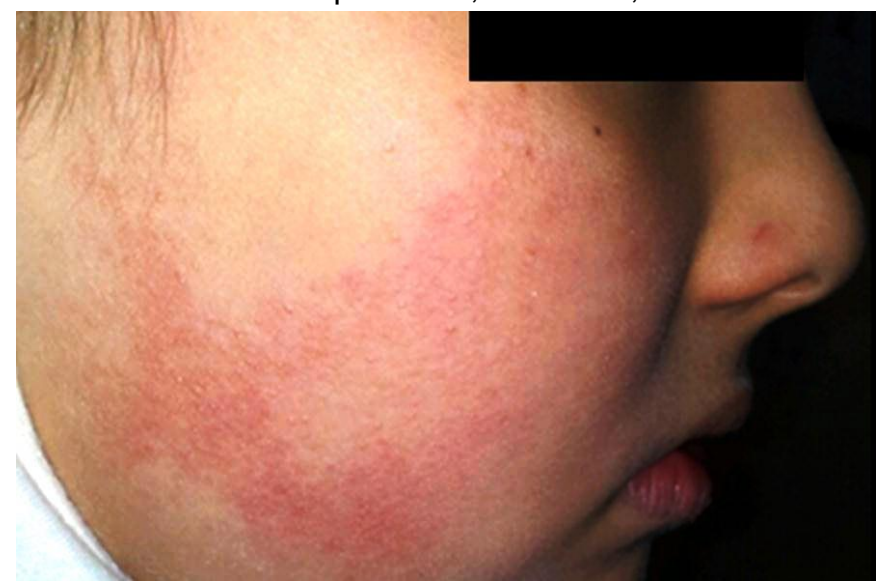

Figure 1. Keratosis pillaris rubra with obvious involvement of the face in 10 years old female patient.

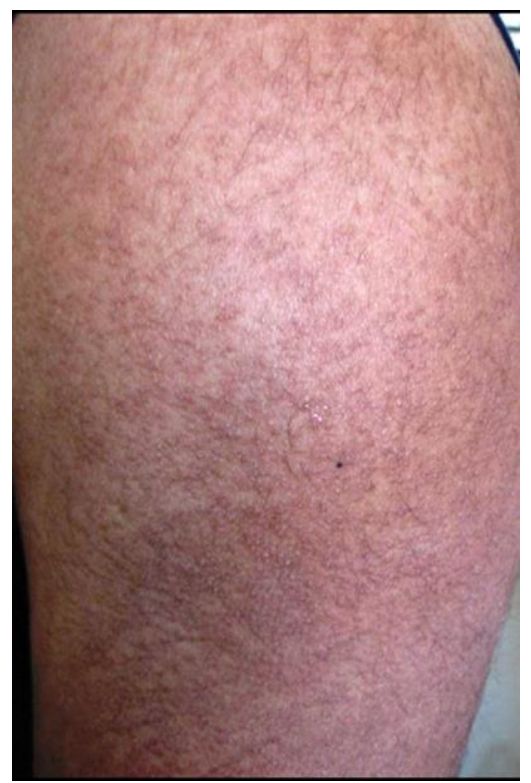

Figure 2. Keratosis pilaris rubra in 28 years old male patient with marked involvement of extensor aspect of the upper arm.

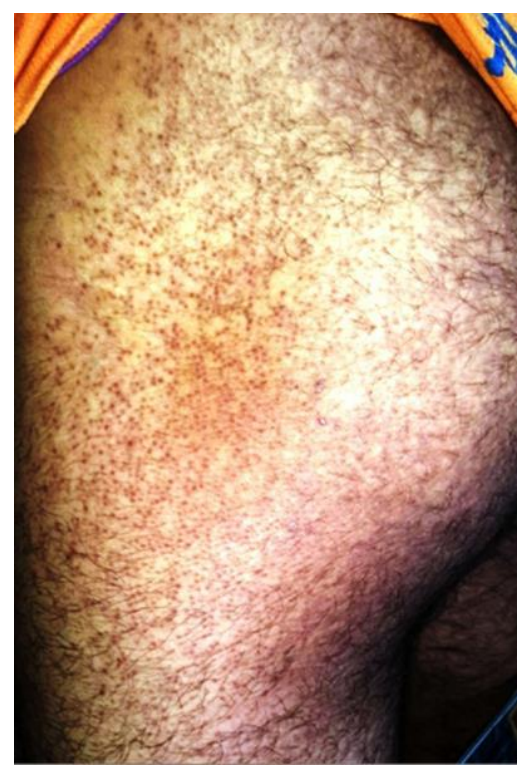

Figure 3. Keratosis pilaris rubra in $\mathbf{3 1}$ years old male patient with recognized involvement of the buttock and thigh. 


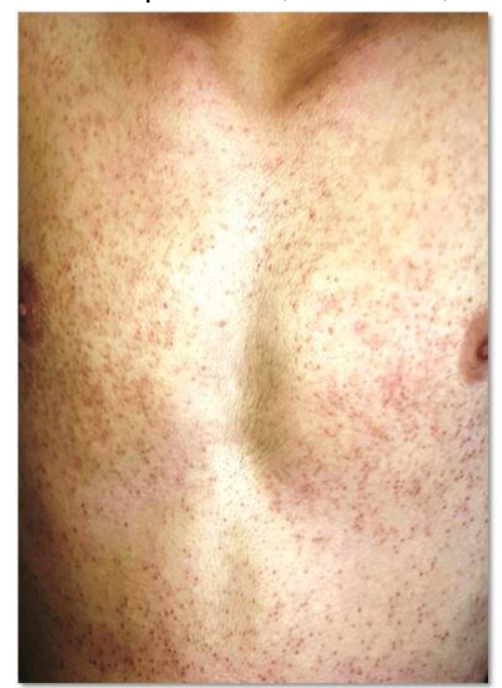

Figure 4. Keratosis pilaris rubra in $\mathbf{2 1}$ years old male patient with marked involvement of trunk.

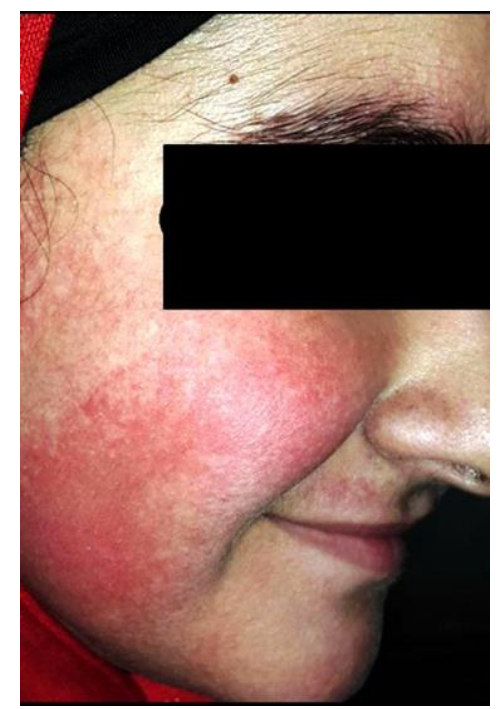

Figure 5. Keratosis pillaris rubra with severe involvement of the face in 17 years old female patient.

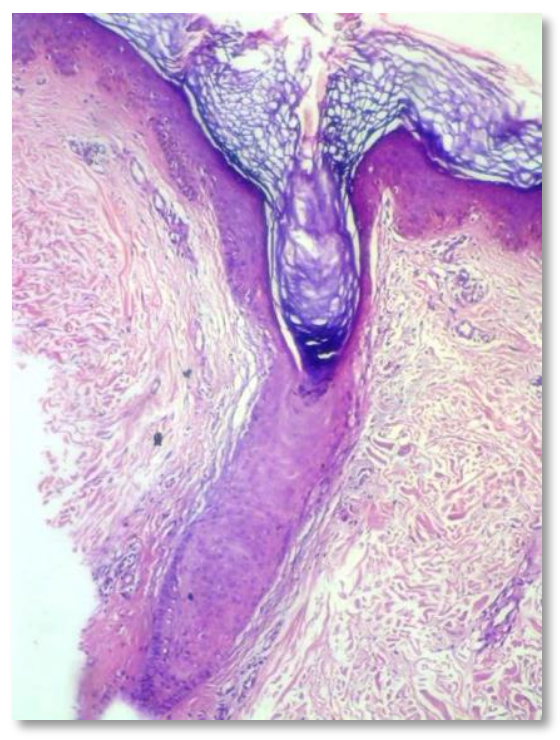

Figure 6. Hematoxylin and Eosin stained section obtained from patient with Keratosis pillaris rubra showing follicular plug fills and dilates infundibulum (x10). 


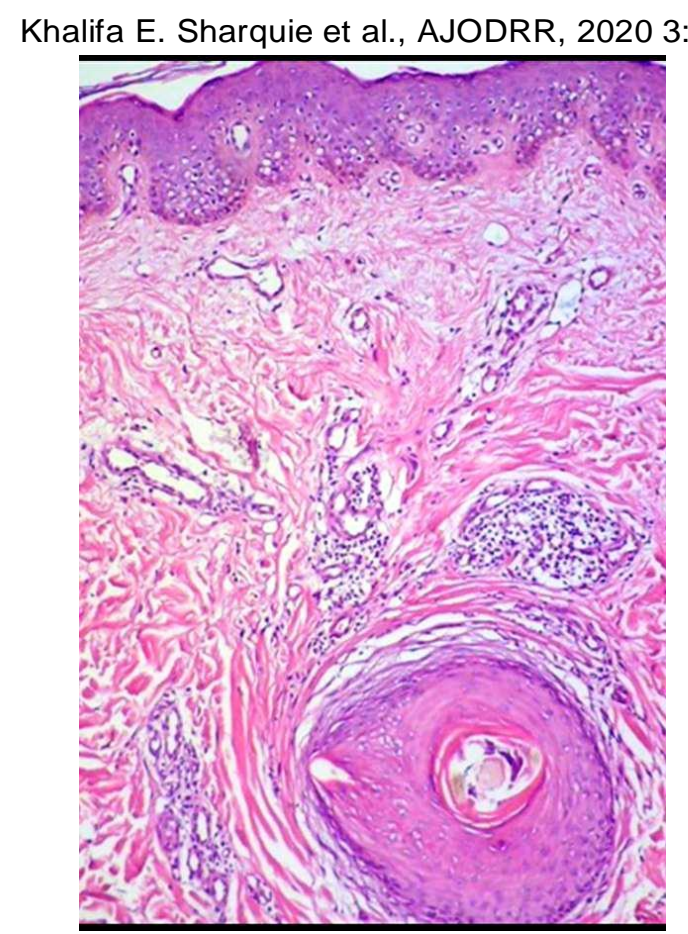

Figure 7. Hematoxylin and Eosin stained section showing dilatation of vascular plexus and lymphocyte infiltration in perivascular and perifollicular area (x10).

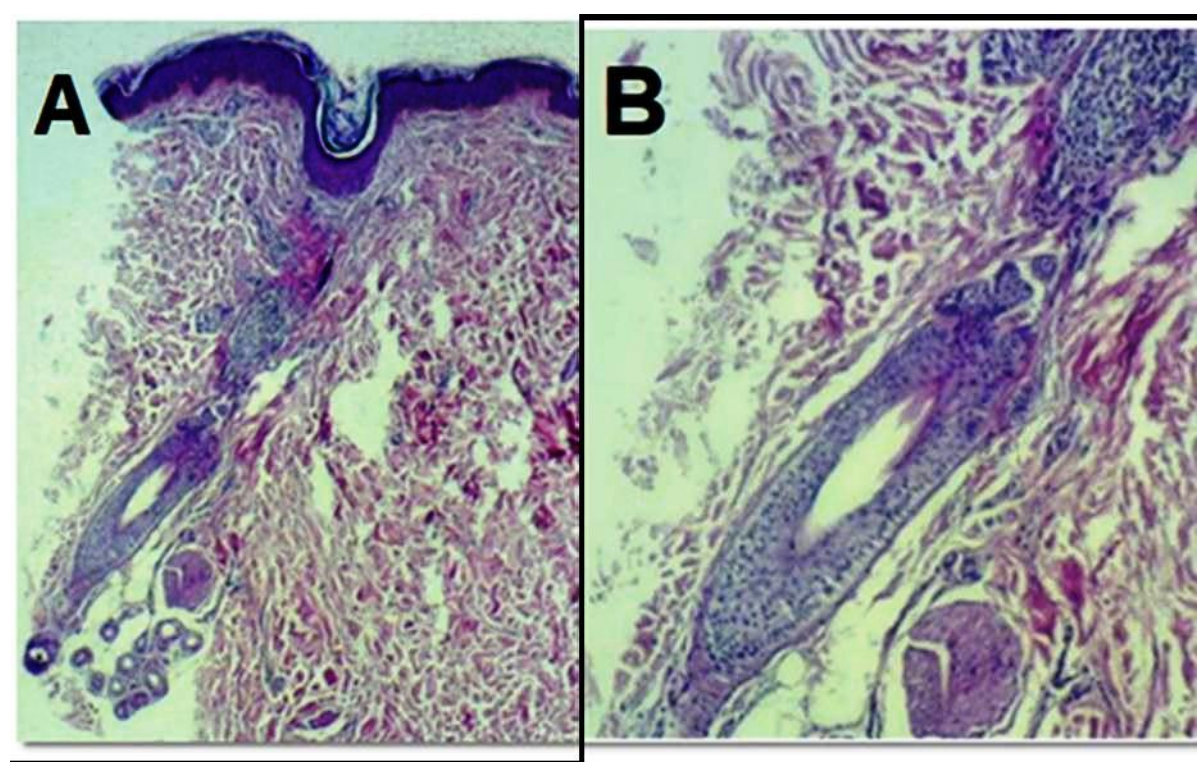

Figure 8. Hematoxylin and Eosin stained sections showing follicular plugging $(A)$ and intrafollicular lymphocytic infiltrates (B). $(A=1 \times 4, B=1 \times 10)$

\section{Discussion}

To the best of our knowledge, this is the first study of KPR in Iraq and even in the world, including large number of cases in a single country (Iraq) showing full clinical and histopathological evaluation of this condition in comparison to Marequeling et al, ${ }^{5}$ case series ,of 27 cases of KPR were reviewed and collected from many countries.
In Marqueling et al case series, $63 \%$ were males and the mean age at onset of disease was 5 years starting mainly during childhood. ${ }^{5}$

The present work showed that the mean age at onset of disease was 2.9 years, $65 \%$ of patients were males and $35 \%$ females. These observations are comparable to that reported by Marqueling et $\mathrm{al}^{5}$, although the mean age at onset was slightly younger, this can be 
explained, in part, by geographic and ethnic variation.

The clinical picture and the morphology of the skin lesion of patients in the present study are similar to the previous reports. ${ }^{5-7}$

Concerning the site of involvement, the present work revealed that facial involvement was in 60 $(100 \%)$ patients , lateral aspects of the arms 60 $(100 \%)$, the thighs including the buttocks in 51 (85\%),the trunk 25 (41.6\%),the legs $22(36.6 \%)$ and the neck $15(25 \%)$. These observations are consistent with Marqueling et al. ${ }^{5}$

Erythema of the side of face and cheeks is the most noticeable feature seen in the present study and often misdiagnosed as dermatitis or butterfly rash of lupus erythematosus.

Erythema of the face in 14 (23.33\%) patients waxed and waned but never completely resolved, while $46(76.66 \%)$ patients showed constant erythema and these observations were not reported in the medical literatures.

Although, the face was the commonly involved site, in addition to extensor surface of the upper and lower limbs, but in many cases the rash extended to involve all hairy areas including the trunk.

The condition was asymptomatic in most patients, but only $8(13,3 \%)$ patients noticed occasional itching which is consonance with Marqueling et al. ${ }^{5}$

In the present study family history with KPR was positive in $8(13,3 \%)$ patients, but unfortunately this finding was not recorded by other published studies to be compared with its result , and this finding does support that a genetic element involved in the etiopathogenesis of this disease.

Most of patients in the present research received several treatments, without substantial improvement. In a minority of patients, improvement of itching and burning sensation but not facial erythema in response to topically applied treatment like steroid or emollients had been observed. While in some patients, topical treatments like urea $10 \%$, retinoids or steroids had been associated with some improvement in the body lesions. Body lesions responded well to treatment with systemic retinoids but no remarkable improvement of facial erythema had been noticed but relapse was occurred on discontinuation of treatment. In many patients, relapse and partial remission in facial and body lesions had been noticed throughout the year with no relation to the season. No other aggravating factors had been recorded.

Many conditions with clinical features that overlap with KPR have been described. The most similar entity to KPR is erythromelanosis follicularis faciei et colli, which is usually seen in adolescents and young adults,commonly in males. Features that differentiate erythromelanosis follicularis faciei et colli from KPR are absence of reported involvement of the trunk and the presence of hyperpigmentation. ${ }^{4}$ Histopathological evaluation showed basket weave appearance with follicular plugging and psoriasiform hyperplasia in few patients. These results are consistent with what has been reported. 6,8 These findings support that this disease has inflammatory elements that is why there is clinically erythema of the cheeks and other parts of the body. The presence of dilated blood vessels may lead to suggestion that pulsed dye laser ( PDL) could be beneficial in reducing erythema particularly of the face and this suggestion has been already established by using PDL as an effective therapy for the erythema associated with KPR especially sides of the face. ${ }^{7}$

In conclusion : Keratosis pilaris rubra is a common condition among Iraqi population and this is the largest study carried out all over the world. It is usually start during infancy, increasing intensity and severity noticed throughout the childhood and adolescence. The main clinical picture was generalized erythematous follicular papules obviously on the face, upper arms, buttocks and thighs in symmetric fashion. Hence, the, name keratosis pilaris rubra totalis is suggested to emphasize the generalized involvement of the disease.

\section{References}

AJODRR: https://escipub.com/american-journal-of-dermatological-research-and-reviews/ 
1. Thomas M, Khopkar US. Keratosis pilaris revisited: is it more than just a follicular keratosis? Int J Trichol 2012; 4:255-8.

2. Hwang S, Schwartz RA. Keratosis pilaris: a common follicular hyperkeratosis. Cutis 2008; 82:177-80.

3. Chu AC, Teixeira F. Acquired Disorders of Epidermal Keratinization. In: Griffiths C, Barker J, Bleiker T, Chalmers R, Creamer D,eds.Rook's Textbook of Dermatology, $9^{\text {th }}$ ed. Singapore, Blackwell Science,2016;87:8-14.

4. Mirmirani $\mathrm{P}$, Rogers M. Keratosis Pilaris and Other Inflammatory Follicular Keratotic Syndromes. In: Goldsmith LA, Kataz SI, Gilchrest BA, Paller SA, Leffell DJ, Wolf $K$, editors. Fitzpatrick's Dermatology in General Medicine, $8^{\text {th }}$ ed. New York, McGrow Hill, 2012; 15, 87:973974.

5. Marqueling AL, Gilliam AE, Prendiville J, Zvulunov A, Antaya RJ, Sugarman $J$,et al.Keratosis pilaris rubra: a common but unrecognized condition. Arch Dermatol 2006; 142:1611-1616.

6. Bukhari I A, Al Ghamdi N, Al Zahrani A, Al Shawarby M. Generalized keratosis pilaris rubra. Our Dermatol Online.2015; 6:187-189.

7. Schoch JJ, Tollefon MM, Witman P, Davis DM. Successful Treatment of Keratosis Pilaris Rubra with Pulsed Dye Laser. Pediatr Dermatol.2016.jul;33:443-6.

8. Weedon D. Disease of cutaneous appendages. In: Weedon's skin pathology, $3^{\text {rd }}$ ed. Edinburgh , Churchil Livi ngstone ,2010;4,15:432-433. 